\section{Ranking data with BASIC}

\author{
JERROLD H. ZAR \\ Northern Illinois University, De Kalb, Illinois
}

Researchers often need to rank data prior to statistical analysis; this is necessary, for example, to employ many nonparametric statistical procedures. The act of ranking assigns consecutive numbers to data to indicate their relative numerical magnitudes. McNally and Wood (1982) presented a BASIC computer program that assigns ranks to all members of a set of data, and when data are tied (i.e., equal in magnitude) assigns to each tied datum the mean of the positions it occupies in the ordered list of data. Ranks could be assigned by first sorting the data; however, the original sequence of data would be lost, which is undesirable, because the original configuration typically must be maintained for statistical and other analyses.

There are better ways to rank data than to sort first, but if sorting is to be performed, it should be by one of many algorithms that have long been known to be more efficient and faster than the "bubble sort" suggested by McNally and Wood (e.g., see Barron \& Diehr, 1983, Cooke, Craven \& Clark, 1982, Knuth, 1973, Loeser, 1974, Lorin, 1975, and Martin, 1971). Lorin (1975) explained that, for $\mathrm{n}$ data, a bubble sort requires a mean of approximately $\left(n^{2} / 2\right)-(3 n / 4)$ comparisons and $n^{2}$ exchanges in a storage array of size $n$. And the better general sorting routines have execution times proportional to $n$ $\log \mathbf{n}$, instead of $\mathbf{n}^{2 .}$ Although the McNally and Wood program does not require preliminary sorting, it appears to be more time consuming than sorting, requiring five passes through the $\mathbf{n}$ data (aside from initializing the arrays and providing output), $2 n^{2}$ comparisons between pairs of data, and a mean of $n+[n(n-1) / 2]$ acts of incrementing elements of the $3 n$ storage matrix.

Listing 1 presents a program that reads in an array of $n$ data and assigns them ranks that are stored in a second array of length $n$ to be displayed as output. The program is written in very elementary BASIC so as to be relatively "portable" among computers. The total number of comparisons required to rank the data is from $n(n-1) / 2$, when there are no ties and the data are submitted in numerical order, to $n(n-1)$, when the data are either in reverse order or all tied. The number of times that members of the array of ranks would be incremented during the ranking process ranges from $n(n-1) / 2$, when there

The author's mailing address: Department of Biological Sciences, Northern Illinois University, De Kalb, IL 60115.

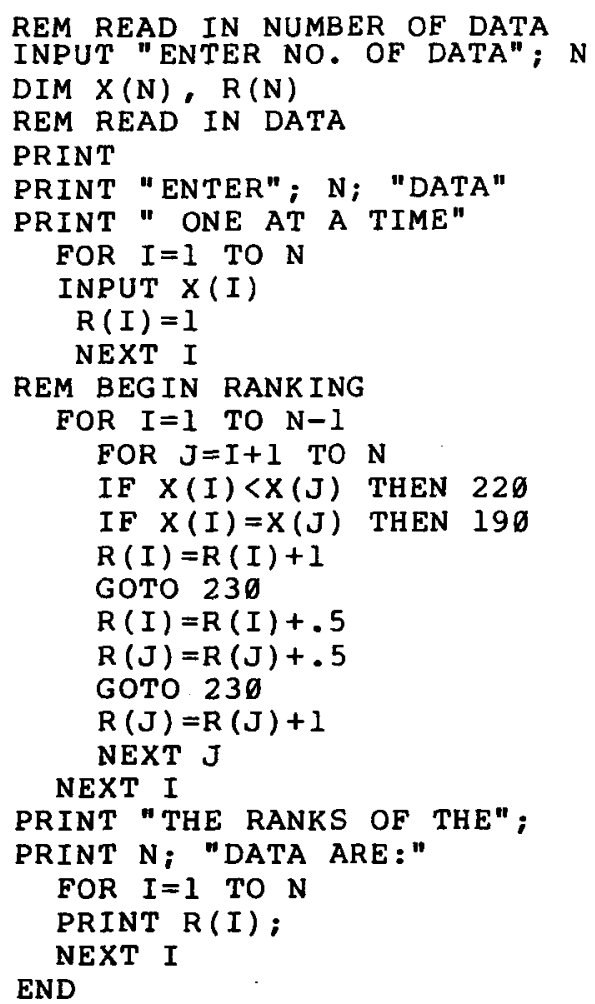

Listing 1. BASIC program for sorting data.

are no ties, to $n(n-1)$, when all the data are tied. Only $2 n$ storage locations are required for the arrays, although a third would likely be needed if the program were modified to compute the "correction for ties" employed by some statistical procedures.

\section{REFERENCES}

BARRoN, T., \& DiEHR, G. (1983, May). Sorting algorithms for microcomputers. BYTE, pp. 482-490.

Cooke, D., Craven, A. H., \& Clarke, G. M. (1982). Basic statistical computing. London: Arnold.

KNUTH, D. E. (1973). The art of computer programming: Vol. 3. Sorting and searching. Reading, MA: Addison-Wesley.

LOESER, R. (1974). Some performance tests of "quicksort" and descendants. Communications of the ACM, 17, 143-152.

LoRIN, L. (1975). Sorting and sort systems. Reading, MA: AddisonWesley.

Martin, W. A. (1971) Sorting. Computing Surveys, 3, 147-174.

MCNALLY, K. K., \& WoOD, D. L. (1982). BASIC rank-order program. Behavior Research Methods \& Instrumentation, 14, 545.

(Revision accepted for publication September 21, 1984.) 